\title{
Telehealth in COVID-19 and Cardiovascular Disease-Ensuring Equitable Care
}

Laureen YT Wang, ${ }^{1} M B B S, M R C P(U K)$, Ting Ting Low, ${ }^{1}{ }_{M B B S, ~ M R C P}(U K)$, Tee Joo Yeo, ${ }^{1}$ MBBS, $M R C P(U K)$

Nine months since the emergence of the SARS-CoV-2 virus, the COVID-19 pandemic has become the largest global public health crisis of the century. With more than a million dead and relentlessly increasing fatalities, the socio-economic consequences are unprecedented. Even cardiovascular disease (CVD), the undisputed leading cause of death worldwide, plays second fiddle to COVID-19. With the limiting of "non-essential" clinical services, the usual delivery of CVD care is significantly hampered-clinic consults are deferred, screening programmes postponed, and rehabilitation classes suspended. ${ }^{1}$ Accordingly, the utilisation of telehealth (the delivery of healthcare services, where patients and providers are socially distanced $)^{2}$ has escalated dramatically, at the risk of leaving marginalised communities behind.

A common denominator in the wars against COVID-19 and CVD is socio-economic inequality, a perennial barrier to infection control and optimal CVD prevention. ${ }^{3}$ Individuals with a lower socio-economic status (SES) account for a higher proportion of COVID-19 infections. ${ }^{4}$ CVD and chronic diseases are more prevalent in these groups and contribute to greater morbidity and mortality with concurrent COVID-19 infection. ${ }^{5}$ To mitigate this, prevention and management of both conditions need to go hand in hand.

Regrettably, low SES populations face substantial difficulty observing public health measures to alleviate COVID-19, whereby (1) crowded living conditions preclude social distancing measures, (2) essential service jobs that cannot be done from home translate to increased exposure to the SARS-CoV-2 virus, (3) limited affordability for tests or treatment restricts healthcare-seeking behaviour, and (4) lifestyles associated with poverty affect nutrition, immunity and overall health, increasing susceptibility to infection. ${ }^{6}$ These challenges mirror those encountered in the fight against CVD, contributing to poor medication compliance, impaired lifestyle modification, reduced access to interventional therapy, and suboptimal cardiac rehabilitation participation.

Singapore, a tiny but highly developed city-state with a population comprising a melange of ethnicities, makes for a compelling case study of telehealth for the dual management of COVID-19 and CVD. Prepandemic, the foundations for telehealth in Singapore were already in place-with up to $82 \%$ of healthcare professionals having prior experience. ${ }^{7}$ Specific to CVD, remote monitoring of blood pressure, weight, blood sugar, as well as teleconsultations for anticoagulation management and troubleshooting of implantable cardiac devices were regularly performed. Dissemination of information via social media, messaging applications and videoconferencing were not uncommon. During the pandemic, centre-based cardiac rehabilitation was not possible and substantial efforts were taken to incorporate telehealth into the delivery of cardiac rehabilitation (e.g. telephone consultations and educational sessions carried out via videoconferencing). In the case of COVID-19, technology facilitated self-checking of symptoms, collection of face masks, directions to COVID-19 ready ambulatory clinics, teleconsultations, and contact tracing. These initiatives, together with strict border control and quarantine measures, led to successful early containment of the disease, drawing global praise. ${ }^{8,9}$ However, an exponential surge of infections within the migrant worker community uncovered a blind spot in Singapore's lauded coronavirus response. ${ }^{10,11}$ Although constituting a fraction of the resident population, migrant workers account for the majority of Singapore's over 58,000 COVID-19 infections to date. Around 300,000 migrant workers live in purpose-built dormitories cramming 10-20 workers per room, and share overloaded communal facilitiesconditions ideal for the spread of COVID-19. Barriers to healthcare accessibility, such as low income, poor health literacy and language differences further compounded

\footnotetext{
${ }^{1}$ National University Heart Centre, Singapore

Address for Correspondence: Dr Tee Joo Yeo, Department of Cardiology, National University Heart Centre Singapore, National University Health System Tower Block, Level 9, 1E Kent Ridge Road, Singapore 119228.

Email: tee_joo_yeo@nuhs.edu.sg
} 
infection risks. Despite Singapore's telehealth capabilities and excellent global rankings in living standards and healthcare, the inordinate impact felt by low SES communities highlights the need for a public health response tailored to vulnerable populations.

Is telehealth the "virtually" perfect solution to optimising COVID-19 and CVD care during the pandemic? ${ }^{10}$ Herein lies a technology "paradox"- those most in need of assistance have the poorest access to technology. ${ }^{12}$ Telehealth-compatible devices or access to the Internet may be unattainable to low-income households, or challenging to adopt by the elderly who are less technologically savvy. Technology's unabated progression adds to an already steep learning curve for those without formal education. Essential workers, such as vocational drivers and cleaners, often have little time for telehealth. Apart from the barriers of time availability and education, there is also the cost of comprehensive services such as vital signs monitoring or electrocardiogram recording. These adjunct technologies provide valuable information in place of physical examination findings, but are often costly and require the user to purchase or loan specialised devices. Rapid telehealth utilisation without improving access, affordability and digital health literacy increases health disparity by bringing upon a new digital divide.
A multi-pronged approach that intervenes at numerous levels is necessary to make telehealth transformative in addressing health disparities (Fig. 1).

Increasing awareness and management of preventive measures for COVID-19 and CVD can be done in parallel, using both traditional media and newer telehealth modalities to improve accessibility to information. Telehealth for the prevention of COVID-19 and CVD should be flexible and applied not only in telephone or video physician consults but also for screening of symptoms, coaching, remote monitoring and allied health support such as nutritional assessments and psychosocial counselling. While embracing telehealth, traditional methods must still be utilised to ensure no one is left out. For instance, flyers, newspapers and magazines featuring health promotion messages can be distributed to the underprivileged. Basic text messages containing reminders to exercise regularly have been shown to improve fitness in patients with CVD, and these may also be applied to emphasise personal hygiene. Longer messages can be pre-recorded and accessed by toll-free telephone numbers. Reinforcement of lifestyle, diet and hygiene measures can be done with radio broadcast or free-to-air television channels.

Besides increasing awareness and preventive management of COVID-19 and CVD, educating and

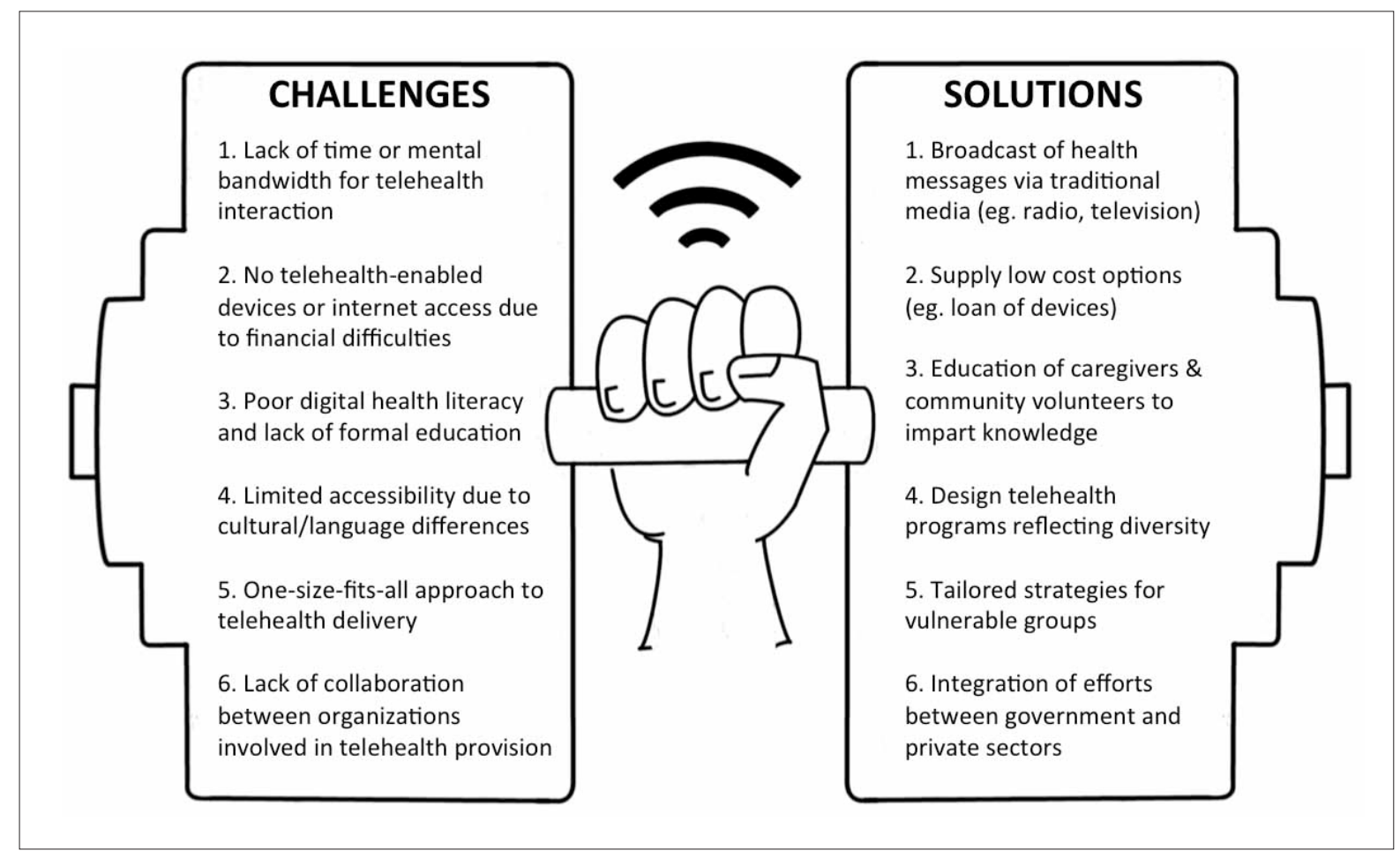

Fig. 1. Equitable Care for COVID-19 and Cardiovascular Disease Through Telehealth 
imparting skills to the community is essential for sustainability of a telehealth programme. Educational sessions on telehealth utilisation can be arranged at community centres, ambulatory clinics, and other areas with high footfall. Trained caregivers and volunteers should be empowered to impart digital knowledge to the vulnerable population. Within local districts, telehealthenabled devices may be provided at no/minimal cost for low-income households. Public and private healthcare institutions should harmonise digital platforms for patient data sharing and facilitate equal access to medical information and telehealth programmes regardless of SES. ${ }^{13,14}$

At the national level, collaboration between governmental ministries, non-governmental organisations and private corporations is critical for financial, logistic and clinical support to enable equitable telehealth. Creating a nationwide framework for widespread health and digital literacy programmes will empower all segments of the population across age groups and SES to take charge of their health. Innovative yet frugal health technologies should take precedence in policy decisions. Overall, policymakers need to acknowledge telehealth as a crucial and cost-effective platform for management of CVD and future infectious disease crises.

The quandaries encountered in managing COVID-19 and CVD are strikingly similar, with vulnerable groups being at a disadvantage. Rapid implementation of telehealth during the pandemic has revolutionised healthcare delivery, but socio-economic inequalities have weakened its impact in the populations who need it most. An overarching series of measures addressing individual barriers, community initiatives and policylevel mindset changes must accompany telehealth advancements. Only then will we be better equipped to provide equitable and sustainable care to all, during a pandemic and beyond.

\section{REFERENCES}

1. Yeo TJ, Wang YL, Low TT. Have a heart during the COVID-19 crisis: Making the case for cardiac rehabilitation in the face of an ongoing pandemic. Eur J Prev Cardiol 2020;27:903-5.

2. World Health Organization. Global Health Observatory (GHO) data Telehealth 2019. Available at: https://www.who.int/gho/goe/telehealth/ en/. Accessed on 7 July 2020.

3. Krishnan L, Ogunwole SM, Cooper LA. Historical insights on coronavirus disease 2019 (COVID-19), the 1918 influenza pandemic, and racial disparities: Illuminating a path forward. Ann Intern Med 2020;173:474-81.

4. Niedzwiedz CL, O’Donnell CA, Jani BD, et al. Ethnic and socioeconomic differences in SARS-CoV-2 infection: prospective cohort study using UK Biobank. BMC Med 2020;18:160.

5. Driggin E, Madhavan MV, Bikdeli B, et al. Cardiovascular considerations for patients, health care workers, and health systems during the COVID-19 pandemic. J Am Coll Cardiol 2020;75:2352-71.

6. Bibbins-Domingo K. This time must be different: Disparities during the COVID-19 pandemic. Ann Intern Med 2020;173:233-4.

7. Philips. Future Health Index 2019. Available at: https://www.philips. com/a-w/about/news/future-health-index/reports/2019/transforminghealthcare-experiences. Accessed on 7 July 2020.

8. Wong JEL, Leo YS, Tan CC. COVID-19 in Singapore-current experience: Critical global issues that require attention and action. JAMA 2020;323:1243-4.

9. Hsu LY, Chia PY, Lim JFY. The novel coronavirus (SARS-CoV-2) epidemic. Ann Acad Med Singap 2020;49:105-7.

10. Sharma M, Scarr S. How migrant worker outbreaks supercharged coronavirus spread in Singapore. Reuters, 22 May 2020. Available at: https://www.reuters.com/article/us-health-coronavirus-singaporeclusters/how-migrant-worker-outbreaks-supercharged-coronavirusspread-in-singapore-idUSKBN22Y29U. Accessed on 7 July 2020.

11. Lim TK. The facts, fallacies and uncertainties about coronavirus disease 2019 (COVID-19). Ann Acad Med Singap 2020;49:343-5.

12. Thomas E, Gallagher R, Grace SL. Future-proofing cardiac rehabilitation: Transitioning services to telehealth during COVID-19. Eur J Prev Cardiol 2020; doi: 10.1177/2047487320922926.

13. Eberly LA, Khatana SAM, Nathan AS, et al. Telemedicine outpatient cardiovascular care during the COVID-19 pandemic: Bridging or opening the digital divide? Circulation 2020; doi: 10.1161/ CIRCULATIONAHA.120.048185.

14. Sittig DF, Singh H. COVID-19 and the need for a national health information technology infrastructure. JAMA 2020;323:2373-4. 